\title{
Support Vector Machines for Anatomical Joint Constraint Modelling
}

\author{
A Dr. Glenn L. Jenkins, Mr. Michael Dacey \\ Swansea Metropolitan University \\ School of Applied Computing \\ Swansea, WALES \\ email: <glenn.l.jenkins $><$ michael.dacey $>@$ smu.ac.uk
}

\begin{abstract}
The accurate simulation of anatomical joint models is becoming increasingly important for both realistic animation and diagnostic medical applications. Recent models have exploited unit quaternions to eliminate singularities when modeling orientations between limbs at a joint. This has led to the development of quaternion based joint constraint validation and correction methods. In this paper a novel method for implicitly modeling unit quaternion joint constraints using Support Vector Machines (SVMs) is proposed which attempts to address the limitations of current constraint validation approaches. Initial results show that the resulting SVMs are capable of modeling regular spherical constraints on the rotation of the limb.
\end{abstract}

Keywords- Support Vector Machine; quaternion; anatomical joint constraint: joint constraint;

\section{INTRODUCTION}

Joint systems are important constituents of anatomical models, they are used in simulation to retain anatomically correct movement and ensure limbs do not separate or intersect. Current techniques are limited by their underlying representation or their abstraction of the joint function. Demand is increasing for anatomically correct joints for applications in animation and medicine $[1,2]$. However in current applications increasing accuracy incurs additional complexity and therefore computational cost [3-5].

Dynamics solutions can be used to produce realistic behavior based on input, contact and constraint forces [6]. Depending on the complexity of the simulation, the outcome of dynamics-based behavior can be difficult to predict. Inverse-Kinematics (IK) based approaches however allow the precise placement of end effectors as constraints [3]. IK solvers attempt to resolve constraints within a constraint system, a problem compounded by the existence of zero or more solutions [3].

Kinematics based solvers can be classified as analytical, often resorting to reduced coordinate formalisms, or numerical, using iterative approaches to solve a system of constraints. An important aspect of this is how the constraint of joints is represented. This work builds on previous work in joint constraint modeling; specifically extending quaternion based phenomenological [7] joints (whose behavior can be modeled without reference to the underlying joint anatomy).
Support Vector Machines (SVMs) are used to implicitly model the boundary between valid and invalid orientation, that is, they learn to classify unit quaternion orientations as valid or invalid. In this paper constraints on the rotation of the limb (or swing [8]) with regular (circular) bounded constrained regions are considered, while irregular boundaries and rotation around the limb (or twist [8]) are the subject of future work.

\section{RELATED WORK}

Primitive joint constraints have been parameterised using Euler angles [9-11]. However inter-dimensional dependencies are not represented [12] and singularities or "Gimbal Lock" are encountered [13]. Inter-dimensional dependencies between Euler angle components can be expressed using equations [14], such equations can provide mathematical descriptions of rotational constraint boundaries. Here geometric functions are fitted to a given dataset, examples include spherical [15] and conical polygons $[1,16]$.

Approaches such as special orthogonal matrices have been used to overcome the problem of singularities $[2,17]$. More recent research has focused on the use of quaternions to model orientations and joint constraints. Quaternion algebra allows rotational models to be represented without the presence of Gimbal Lock [13].

Quaternions are an extension of complex numbers, composed of one real and three imaginary components where $\mathrm{q}=\langle\mathrm{s}, \mathrm{i}, \mathrm{j}, \mathrm{k}\rangle$. Multiplying complex numbers results in rotation in the complex plane, giving rise to the complex identity $i^{2}=-1$. This is extended in a subset of quaternion space, where all quaternions are of unit length, to $i^{2}=j^{2}=k^{2}$ $=-1$. Unit quaternions occupy a three dimensional surface $(\mathrm{a}$ hyper-sphere) in four dimensional space and can be used to represent rotations. This representation is redundant as the unit quaternions represent $4 \pi$ rotations, hence equations at polar opposites $(\mathrm{q}$ and $-\mathrm{q})$ represent the same rotation [18] i.e. they are antipodal.

Lee [19] decomposes a single unit quaternion into two unit quaternions each representing rotation in a single plane (effectively swing and twist for conic and axial constraints). Conic, axial and revolute constraints are defined and more complex constraints can modelled with a union of these basic types. Interrogation of these shapes (to ascertain the validity of a joint configuration,) is presented, but no method of calculating a correction to the nearest valid orientation is defined. Liu and Prakash [20] build on Lee's work. Using a 
sampled boundary they create a function to constrain the decomposed quaternion that can be used for both constraint validation and clamping to the boundary.

In the quaternion iso-surface approach of Herda et al [21, 22] limb rotations were recorded and represented in quaternion space. A set of four-dimensional unit quaternions describing the valid joint rotations are projected to a cloud of points in three-dimensions. This reduction in dimensionality overcomes the problem of ambiguity in quaternion space and allows the creation of an iso-surface surrounding the cloud of valid points defining the boundary between valid and invalid rotations. An iterative approach can then be employed to resolve invalid joint configurations.

Johnson [23] also reduced the dimensionality of the quaternion by projecting one half of the unit quaternion hyper-sphere onto a three-dimensional tangent space. A set of quaternions expressing valid joint and pose constraints are generated and constraints implemented based on a maximum deviation from their mean. Corrections are implemented by recursively moving an invalid point closer to the mean and the corrected point is then mapped back into unit quaternion space.

In recent work the author has successfully evolved and trained Generalised Multi-layer Perceptron Neural Networks to provide a suitable correction quaternion based on the input orientation with a zero correction for valid points [24, 25]. Unlike the approaches of the other authors [19-22, 26] this approach does not require pre-processing of the subject quaternion. However, due to error in the neural network some correction of valid orientations takes place, which we hope to overcome using SVMs.

The SVM attempts to identify a hyper-plane that separates two groups of data at maximum distance from a select number of input vectors (known as support vectors). Support Vector Machines (SVMs) rely on the principle of structural risk minimisation (SRM) [27]. Training of the machines aims to minimise both error and network complexity maintaining generalisation capabilities [28]. Unlike traditional neural network training which attempts to solve a nonconvex unconstrained minimisation problem, the SVM minimises both learning machine error and complexity by solution of a quadratic programming problem with linear constraints [28, 29].

Where the two groups are not linearly separable, slack variables are introduced that relax the constraints governing the distance of the hyper-planes from the support vectors with the penalty of further cost [29]. Where a nonlinear separation exists SVMs capitalise on the only reference to the training data in the in the optimisation of the hyper-plane being through a dot product. A kernel function is used which maps the data to some other possibly infinite Euclidean space. The choice of kernel function has an affect the performance of the SVM and is problem dependent [30].

SVMs may be capable of overcoming the limitations of existing quaternion joint constraint validation approaches [19-22, 26] and complementing corrective approaches [24]. The SVM may be capable of processing quaternions without decomposing the quaternion to an axis-angle representation
$[19,20]$ or mapping the quaternion to a three-dimensional space $[21,22,26]$.

In terms of modelling a virtual limb current approaches are capable of modelling regular boundaries [19, 26] and irregular boundaries [20-22] between valid and invalid limb orientations (relative to an attachment point). SVMs should in theory be able to model both regular and irregular boundaries. Current approaches also model the rotation around the limb [19-22, 26]. In this paper we begin to explore the capabilities of SVMs in modelling such constraints. Limited to regular rotational boundaries with no constraint on the rotation around the limb, the aim of this initial study is to complement the techniques developed in our earlier work on corrective neural network constraints $[24,25]$.

The remainder of this paper is structured as follows. Section III provides a description of our methodology with reference to the techniques employed. Section IV outlines the experiments undertaken and the results obtained, Section IV discuss and Second $\mathrm{V}$ draws conclusions from these results.

\section{METHODOLOGY}

This paper describes the application of SVMs to the classification of unit quaternions describing the orientation of an anatomical limb. In doing so the SVM implicitly learns the boundary between valid and invalid orientations in unit quaternion space.

SVMs were trained to classify valid and invalid orientations for constraints of various sizes to ascertain their performance in the context of anatomical models. In each case linear, polynomial, RBF and sigmoidal kernel functions were applied in turn to identify the most suitable.

An SVM was trained using a dataset based on the simple model shown in Fig. 1. The angle between a random vector (virtual limb) and the $\mathrm{z}$-axis was used constrained to a given angle (in degrees). The orientation of the vector (as a unit quaternion) and validity of the orientation (as a group number) form the training data. This effectively gives a two dimensional boundary on the surface of a three dimensional sphere, similar to the boundaries modelled by other authors $[15,19,24]$.

While training neural networks in earlier work [25] the authors found that valid regions on both sides of the hypersphere (both $\mathrm{q}$ and its antipode $-\mathrm{q}$ being valid) disrupted learning. In the training set all valid patters are placed one side of the hyper-sphere and all other patterns are considered invalid, this fits the context of anatomical constraints as a rotation though $360^{\circ}$ should not be valid. 


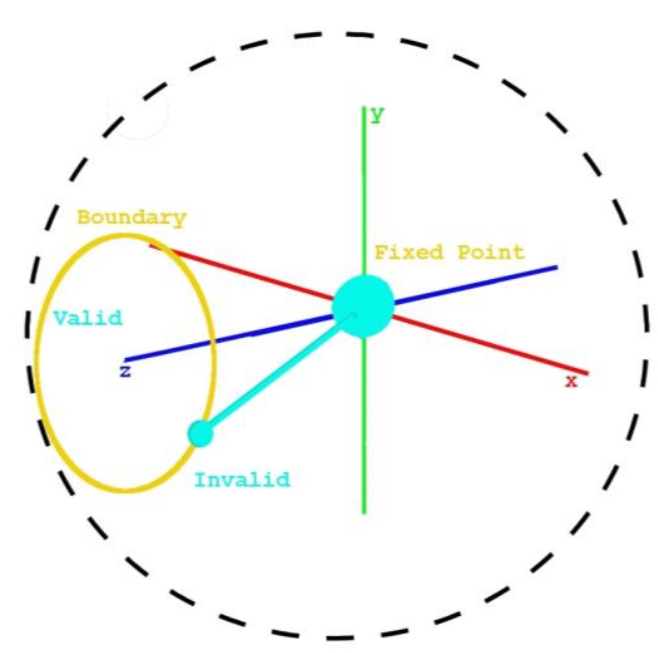

Fig. 1 - Model used for dataset generation. Valid region inside boundary invalid region outside boundary

For each of the experiments five hundred patterns were used evenly distributed between the valid and invalid regions. Two datasets were created for each experiment, the training set was used to train the SVM and the test set provided measurement of the SVMs generalisation capabilities.

The SVM used in this work is SVMLight [31] a state of the art SVM implementation developed by T. Joachims who has published substantial material on improving the performance of the SVM [31-34].

\section{RESULTS}

The results show that the SVM is able to classify the joint orientations as valid or invalid with a high degree of accuracy. The sigmoid kernel function performs poorly with a maximum correct classification of less than $60 \%$. The results for linear, polynomial and radial basis kernel functions demonstrate higher performance above $90 \%$ for all ranges. The results for nonsigmoidal kernel functions share a similar distribution and are almost symmetrical around ninety degrees (angle between virtual limb and z-axis). These results are shown in Fig. 2.

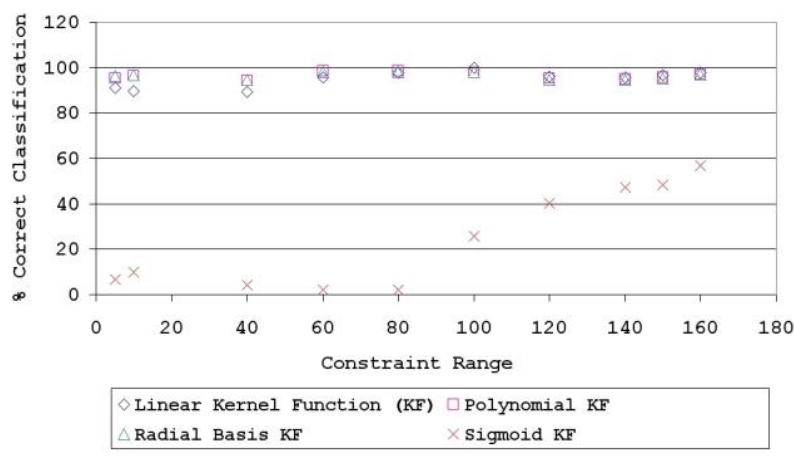

Fig. 2 - Performance of the SVM on a quaternion based constraint with a regular boundary.
Improvements in performance were attempted by increasing the number of patterns used in training. A constraint of twenty degrees radius was selected as the results showed scope for improvement. Increasing the number of training patterns improves performance in the case of the linear, polynomial and radial basis kernel functions, but not in the case of the sigmoid function. The increases in performance attenuate with the increase in training patterns, hence the benefits of increasing the number of patterns are negligible above a threshold.

\section{DISCUSSION}

The results show that SVMs are capable of classifying unit quaternions representing the orientations of a virtual anatomical limb as either valid or invalid. Hence they implicitly model the boundary between valid and invalid orientations in unit quaternions space. The linear kernel performs very well, indicating that the majority of the data is linearly separable in unit quaternion space. This indicates that the valid and invalid regions are almost linearly separable. Both the RBF and polynomial kernels perform better than the linear kernel which is encouraging for future work with more complex boundaries that may not be linearly separable in unit quaternion space. The sigmoidal kernel function performs poorly on all ranges.

The performance of linear, RBF and polynomial kernel functions is good regardless of the size of the constrained region. There is some symmetry around ninety degrees returning to the model it is clear that the boundary in this case is the diameter of the unit sphere traced by the virtual limb, hence the boundaries of the constraints on either side are of equal size. This performance increase is ascribed to the distribution of patterns, which would be most even when the sizes of the valid and invalid regions were equal.

Forcing valid orientations to one side of the unit quaternion hyper-sphere reduces the complexity of the separation and introduces a limitation to the use of this technique to classify any rotation. A valid rotation on the opposite side of the hyper-sphere would be reported as invalid. This is not a limitation in the context of anatomical constraints provided that the initial joint configuration is on the appropriate side of the hyper-sphere. The constraint correction (or clamping) system should ensure that the orientation remains within the boundary and prevent the limb from performing a full rotation to another valid configuration.

SVMs may be capable of classifying orientations in quaternion space without enforcing a single valid region, however SVMs are binary classifiers. A popular solution is to decompose the multi-class classification problem to multiple binary problems [35]. Recently the inclusion of binary tree structures [35], directed acyclic graphs [36] and other methods have been suggested [36]. Utilization of such techniques to classify orientations in unit quaternions space with multiple valid regions may form a part of future research.

Other limitations include the choice of kernel function which often relies on trial and error [29] this choice is limited to functions which obey Mercer's conditions [37]. 
The computational cost of training is high and although this been successfully reduced issues with scalability exist [29, 31, 38]. The quadratic programming problem central to SVM training is usually complex and subject stability problems [38], reformulation has been attempted to reduce complexity and improve stability [38]. During training SVMs may select sub-optimal support vectors for categories within the training set, multi-pass systems that identify the best candidates for support vectors prior to SVM training have been developed [39].

\section{CONCLUSIONS AND FUTURE WORK}

In conclusion SVMs are capable of implicitly modelling the boundary between valid and invalid orientations in quaternion space to a reasonable degree of accuracy. More importantly they can classify valid and invalid quaternion based orientation constraints like those suggested by Lee [19] and Johnson [26] to a very high degree of accuracy. Unlike these approaches no decomposition or reformatting of the unit quaternion orientation is required.

SVMs are extremely capable classifiers which using kernel functions map the input domain into an alternative Euclidean space. They may be capable of delineating the boundary between two groups of orientations (valid and invalid) in quaternion space for a more general case with valid regions on both hemispheres of the quaternion hypersphere. Alternatively additional groups may be provided (for example an additional valid group,) allowing successful classification of orientations on either side of the hyper sphere. This will be investigated in future work.

There is some evidence that SVMs may cope well with irregular boundaries and may offer advantages over the current approaches of Herda et al [21, 22] and Liu and Prakash [20]. There is also the subject of rotation around the limb which has not been considered here. Both are topics for future research.

\section{ACKNOWLEDGMENT}

The authors would like to thank Thorsten Joachims (Cornell University,) for providing the SVMlight software and our colleagues at Swansea Metropolitan University for their continued support and advice.

\section{REFERENCES}

[1] W. Manurel and D. Thalmann, "Human Shoulder Joint Modelling Including Scapulo-Thoratic Constraints and Joint Sinus Cones," Computers and Graphics, vol. 24, 2000, pp. 203-218.

[2] J. D. Feikes, J. J. O'Connor, and A. B. Zavatsky, "A Constraint-Based Approach to Modelling the Mobility of the Human Knee Joint," Journal of Biomechanics, vol. 36, 2003, pp. 125-129.

[3] A. Watt and M. Watt, "Forward Vs Inverse Kinematics in Computer Animation," in Advanced Animation and Rendering Techniques. New York: ACM Press, 1992, pp. 371-384.

[4] Y. Zhang and J. Wang, "A Dual Neural Network for Constrained Torque Optimization of Kinematically Redundant Manipulators," IEEE Transactions on System, Man and Cybernetics: Part B, vol. 32, 2002, pp. 654-662.

[5] A. D'Souza, V. S, and S. Stefan, "Learning Inverse Kinematics," Proc. International Conference on Intelligent Robots and System, Maui, Hawaii, USA, 29 Oct - 3 Nov 2001, pp. 298-303.
[6] P. M. Issacs and F. C. Micheal, "Controlling Dynamic Simulation with Kinematic Constraints, Behaviour Functions and Inverse Dynamics," ACM Transactions on Computer Graphics, vol. 21, 1987, pp. $215-223$.

[7] A. E. Engin and S. T. Tumer, "Improvised Dynamic Model of Human Knee Joint and Its Response to Loading," Journal of Biomechanical Engineering, vol. 115, 1993, pp. 137-142.

[8] F. S. Grassia, "Practical Parameterization of Rotations Using the Exponential Map," Journal of Graphics Tools, vol. 3, 1998, pp.

[9] J. J. Faraway, X. Zhang, and D. B. Chaffin, "Rectifying Postures Reconstructed from Joint Angles to Meet Constraints," Journal of Biomechanics, vol. 32, 1999, pp. 733-736.

[10] J. Eng and D. A. Winter, "Kinematic Analysis of the Lower Limbs During Walking: What Information Can Be Gained from a $3 \mathrm{~d}$ Model," Journal of Biomechanics, vol. 28, 1995, pp. 753-758.

[11] T. Furuta, T. Tawara, Y. Okumura, M. Shimizu, and K. Tomiyama, "Design and Construction of a Series of Compact Humanoid Robots \& Development of Bipedal Walking Control Strategies," Robotics and Autonomous Systems, vol. 37, 2001, pp. 81-100.

[12] P. Baerlocher, "Inverse Kinematics Techniques for the Interactive Posture Control of Articulated Figures," Ecole Polytechnique Federal De Lausanne, Lausanne, $\mathrm{PhD}$ thesis, 2001.

[13] A. Watt and M. Watt, "Parameterisation of Rotation," in Advanced Animation and Rendering Techniques. New York: ACM Press, 1992, pp. 356-368.

[14] A. Maciel, L. P. Nedel, and C. M. Freitas, "Anatomy Based Joint Models for Virtual Human Skeletons," Proc. IEEE Computer Animation, Geneva, Switzerland., 19 - 21 June 2002, pp. 220-224.

[15] J. U. Korein, A Geometric Investigation of Reach. Massachusetts: MIT Press, 1984.

[16] A. E. Engin and S. T. Tumer, "Three Dimensional Kinematic Modelling of the Human Shoulder Complex Part 1: Physical Model \& Determination of Joint Sinus Cone," Journal of Biomechanical Engineering, vol. 111, 1989, pp. 107-112.

[17] D. R. Wilson, J. D. Feikes, and J. J. O'Connor, "Ligaments and Articular Contact Guide Passive Knee Flextion," Journal of Biomechanics, vol. 31, 1998, pp. 1127-1136.

[18] A. Hanson, "Fundamentals of Rotation," in Visualising Quaternions, T. Cox, Ed. San Francisco, CA, USA: Morgan Kaufmann, 2006, pp. 43-56.

[19] J. Lee, "A Hierarchical Approach to Motion Analysis and Synthesis for Articulated Figures," Korean Advanced Institute of Science and Technology, Daejeon, PhD thesis, 2000.

[20] Q. Liu and E. Prakash, C, "The Parameterization of Joint Rotation with the Unit Quaternion," Proc. 7th Digital Image Computing: Techniques and Applications, Sydney, 10-12 December 2003, pp. 410-417.

[21] L. Herda, R. Urtasun, P. Fua, and A. Hanson, "Automatic Determination of Shoulder Joint Limits Using Quaternion Field Boundaries," International Journal of Robotics Research, vol. 22, June 2003, pp. 419-444.

[22] L. Herda, R. Urtasun, and P. Fua, " Hierarchical Implicit Surface Joint Limits for Human Body Tracking," Computer Vision Lab, Ecole Polytechnique Federal de Lausanne (EPFL), Lusanne CH-1015, 2004.

[23] M. P. Johnson, "Exploiting Quaternions to Support Expressive Interactive Character Motion," Massachusetts Institute of Technology, Massachusetts, PhD thesis, 1995.

[24] G. Jenkins and P. Angel, "Evolved Topology Genralized Multi-Layer Percerptron (Gmlp) for Joint Constraint Modelling," Proc. 9th International Conference on Computer Modelling and Simulation, United Kingdom Simulation Society, Oriel Collage Oxford, 4-6th April 2006.

[25] G. Jenkins, "Evolved Neural Network Approximation of Discontinuous Vector Fields in Unit Quaternion Space $\left(S^{3}\right)$ for Anatomical Joint Constraint," University of Glamorgan, Treforest, $\mathrm{PhD}$ thesis, 2007. 
[26] M. P. Johnson, "Exploiting Quaternions to Support Expressive Interactive Character Motion," Massachusetts Institute of Technology, Massachusetts, PhD thesis, 2003.

[27] V. Vapnik, The Nature of Statistical Learning Theory, 2 ed. New York: Springer, 1995.

[28] A. Autret, "Modular Neural Networks for Analysis of Flow Cytometry Data," University of Glamorgan, Treforest, PhD thesis, 2003.

[29] C. J. C. Burges, "A Tutorial on Support Vector Machines for Pattern Recognition," Data Mining and Knowledge Discovery, vol. 2, 1998, pp. 121-167.

[30] V. Vapnik, S. E. Golowich, and A. J. Smola, "Support Vector Method for Function Approximation, Regression Estimation and Signal Processing," Advances in Neural Information Processing, vol. 9, 1997, pp. 281-287.

[31] T. Joachims, "Making Large-Scale Svm Learning Practical," in Advances in Kernel Methods - Support Vector Learning, B. Scholkopf, C. J. C. Burges, and A. J. Smola, Eds. Cambridge: MIT Press, 1999.

[32] T. Joachims, "Making Large-Scale Svm Learning Practical," Universitat Dortmund, Dortmund, LS, LS8-Report 24, 1998.

[33] T. Joachims, "Transductive Inference for Text Classification Using Support Vector Machines," Proc. International Conference on Machine Learning, Morgan Kaufmann Publishers Inc, Bled, Slovenia, 27 - 30 June 1999, pp. 200-209.

[34] T. Joachims, "Estimating the Generalization Performance of a Svm Effciently," Proc. 17th International Conference on Machine Learning, Morgan Kaufman, Stanford, USA 2000, pp. 431-438.

[35] S. Cheong, S. H. Oh, and S. Lee, "Support Vector Machines with Binary Tree Architeture for Multi-Class Classification," Neural Information Processing, vol. 2, 2004, pp. 47-51.

[36] P. Mahesh, "Multiclass Approaches for Support Vector Machine Based Land Cover Classification," Proc. MapIndia, Hotel Taj Palace, New Delhi, 7-9 February 2005.

[37] N. Herandez, I. Talavera, A. Dago, R. J. Biscay, M. M. Castro Fereria, and D. Porro, "Relevence Vector Machines for Multivariate Calibration Purposes," Journal of Chemometrics, vol. 22, 2008, pp. 686-694.

[38] S. Vijayakumar and S. Wu, "Sequential Support Vector Classifiers and Regression," Proc. International Conference on Soft Computing, 1999, pp. 610-619.

[39] T. Masuyama and H. Nakagawa, "Two Step Pos Selection for Svm Based Text Categorization," IECE Transactions on Information and Systems, vol. E87-D, February 2004 2004, pp. 1-7. 